Archives

\title{
« Par la volonté du Duce et par l'œuvre du Parti »
}

Le mythe du chef dans le Guide historique de l'Exposition de la Révolution fasciste

\section{Maddalena Carli}

\section{(2) OpenEdition}

12 Journals

\section{Édition électronique}

URL : http://journals.openedition.org/ccrh/301

DOI : 10.4000/ccrh.301

ISSN : 1760-7906

Éditeur

Centre de recherches historiques - EHESS

\section{Édition imprimée}

Date de publication : 30 avril 2003

ISSN : 0990-9141

\section{Référence électronique}

Maddalena Carli, « «Par la volonté du Duce et par l'œuvre du Parti » », Les Cahiers du Centre de Recherches Historiques [En ligne], 31 | 2003, mis en ligne le 15 septembre 2008, consulté le 10 décembre 2020. URL : http://journals.openedition.org/ccrh/301 ; DOI : https://doi.org/10.4000/ccrh. 301

Ce document a été généré automatiquement le 10 décembre 2020.

Article L.111-1 du Code de la propriété intellectuelle. 


\section{« Par la volonté du Duce et par l'œuvre du Parti »}

Le mythe du chef dans le Guide historique de l'Exposition de la Révolution fasciste

Maddalena Carli

\section{NOTE DE L'AUTEUR}

Cet article présente une partie des problématiques de ma thèse « Voir le fascisme ».

Expositions d'un régime totalitaire (1932-1942), dirigée par le professeur Christophe

Prochasson.

$1 \quad$ Les dictateurs de l'entre-deux-guerres sont au centre des analyses et de la réflexion des historiens dès les années cinquante. Non seulement dans le domaine de la comparaison entre régimes totalitaires mais aussi dans la perspective des histoires nationales : il suffit de rappeler - pour ne s'en tenir qu'aux cas allemand et italien - d'une part que l'évaluation du rôle joué par Adolf Hitler dans l'édification de l'état nazi fut à l'origine de la division entre «intentionnalistes » et «fonctionnalistes » qui caractérisa, et qui marque encore, les études sur le Troisième Reich ${ }^{1}$; et d'autre part qu'autour de l'itinéraire politique et intellectuel de Benito Mussolini fut conçue la principale entreprise historiographique sur le régime fasciste : la monumentale biographie du duce publiée - entre 1965 et 1990 - par Renzo De Felice ${ }^{2}$.

2 Simultanément impliquée dans les évolutions de la littérature critique sur les totalitarismes et dans le renouvellement du genre biographique ${ }^{3}$, la question du leadership suggère à présent des réflexions nouvelles. L'ouverture de la recherche historique aux aspects sociaux et culturels des systèmes politiques, et l'attention prêtée à leur fonctionnement symbolique ont permis de considérer les dictateurs comme auteurs et comme objets de véritables «religions laïques $»^{4}$. Les études sur la construction du consensus se sont néanmoins orientées presque exclusivement vers l'examen des rituels - et des décors architecturaux et monumentaux qui en permettaient le déroulement - 
inspirés par le culte du chef. Elles se ressentent de l'absence d'une dimension spécifiquement consacrée aux formes et aux fonctions des images, et au changement de paradigme dont elles furent l'objet dans l'Europe de la première moitié du $\mathrm{xx}^{\mathrm{e}}$ siècle ; une modification qui est profondément liée à la naissance des moyens modernes de communication et de propagande, et aux transformations introduites - dans l'élaboration doctrinale et dans les comportements politiques - par la réalisation des processus de nationalisation des masses.

Il suffit de rappeler le traitement réservé aux expositions fascistes, largement examinées dans leurs implications esthétiques mais presque ignorées en tant qu'instruments de la médiation culturelle. Soumise à une profonde réorientation stylistique, la politique d'exposition du fascisme commença, au début des années trente, à s'éloigner du canon des rendez-vous artistiques consacrés - la Biennale de Venise et la Quadriennale de Rome avant tout - et à promouvoir des manifestations thématiques qui offraient des espaces originaux et des possibilités exceptionnelles à la narration par images. Les historiens américains parlent, à cet égard, d'une attitude " exhibitionniste » du régime, qui visait à augmenter sa visibilité interne et internationale, et à promouvoir les projets de régénération implicites dans sa vocation totalitaire ${ }^{5}$. Dans les pages qui suivent, je voudrais proposer quelques réflexions sur l'événement qui inaugura, et réalisa intégralement, ce modèle d'exposition : l'Exposition de la Révolution fasciste (Mostra della Rivoluzione fascista) $)^{6}$, ouverte au public le 28 octobre 1932 et proposée à nouveau - en vertu d'un processus de muséification qui eut tendance à la transformer en institution permanente - à l'occasion du Bimillénaire de la naissance d'Auguste (1937) et des célébrations pour le vingtième anniversaire de la prise du pouvoir (Ventennale, 1942). Parmi les nombreuses initiatives destinées à en propager l'existence, une place importante fut tenue par la publication d'un Guide historique (1933), se composant d'une introduction à la période exposée (éloquemment intitulée " Mussolini et la Révolution ») et d'une description de l'itinéraire historico-artistique ${ }^{7}$. Euvre de facture étonnante, où, comme pour recréer l'interaction entre documents et images de la manifestation, les mots des auteurs cohabitent avec les reproductions photographiques du montage, le Guide de l'exposition représente une observatoire privilégié non seulement de la pluralité de langages qui concoururent à la fabrication du charisme du chef mais aussi de la polysémie de ce dernier, et des liens qu'il noua avec les symboles restants du pouvoir totalitaire : les mythes des origines révolutionnaires et de l'homme nouveau.

\section{"Les vainqueurs, par leur victoire, acquièrent le droit d'écrire l'histoire »}

4 Annoncé - « dans l'attente de publier un catalogue analytique complet et détaillé » - par une brochure conçue par la présidence de l'Exposition (1932) ${ }^{8}$ et réimprimé, en 1982, par l'hebdomadaire de la nouvelle droite Candido Nuovo dirigé par Giorgio Pisanò, le Guide historique de 1933 a accompagné l'Exposition de la Révolution fasciste bien après les dix années de sa durée effective. Point de repère pour le public de l'époque, il a participé de la postérité de l'événement, en nourrissant la mémoire nostalgique des milieux néofascistes et, en même temps, les activités de recherche: face aux lacunes de la documentation d'archives, les images, les commentaires et les informations du Guide ont représenté l'une des sources privilégiées par les spécialistes de l'art et de la culture fascistes. 
5 À en parcourir les pages, on constate que le guide restitue toujours l'impact suscité par la majesté de l'Exposition, et la nature " modernissima », « hardie », d'un décor qui présente plus qu'un point de contact avec l'œuvre d'art totale d'inspiration avant-gardiste, en vertu des techniques employées et de la conception de l'espace véhiculée. Les textes du Guide présentent en outre de nombreux indices des raisons qui décidèrent la dictature italienne à la mise en scène de ses propres origines, à savoir « du but, du caractère et de la signification de l'exposition » à laquelle le régime confia la représentation et la mémoire de son propre passé révolutionnaire. Avant d'examiner le volume en question, il me semble toutefois nécessaire de mettre en relation la manifestation de 1932 avec le parcours s'étendant sur plus de dix ans qui fit de l'Exposition de la Révolution fasciste un événement et une institution de l'univers culturel totalitaire.

6 La proposition d'organiser une exposition pour célébrer le dixième anniversaire des Faisceaux de combat - le mouvement politique fondé par Benito Mussolini le 23 mars 1919 afin de "perpétuer l'esprit interventionniste en période de paix »- fut avancée par le président de l'Institut fasciste de la culture de Milan, Dino Alfieri, en février $1928^{\circ}$. En juin un Comité de direction, auquel participaient les plus hautes autorités du Parti national fasciste (Pnf) vit le jour et fut chargé de la mise en place de la manifestation et du repérage du matériel documentaire ${ }^{10}$. Il fallut quatre ans et de nombreuses modifications au programme originel pour que le projet aboutisse. En mettant fin à une phase durant laquelle l'idée sembla être définitivement abandonnée, une loi promulguée par le Grand Conseil dans sa session du 14 juillet 1931 relançait l'idée d'une exposition du fascisme; elle en confiait l'organisation à Dino Alfieri et à un groupe restreint de collaborateurs (Luigi Freddi, Alessandro Melchiorri, Cipriano Efisio Oppo, Ambrogio Devoto), fixait son ouverture au 28 octobre 1932 et transférait son siège de Milan au Palais des expositions de la capitale ${ }^{11}$. En vertu du nouveau plan, la manifestation aurait dû se développer autour de quatre thèmes - l'État, le travail, les armes et l'esprit - et polariser la narration sur la période comprise entre la fondation des Faisceaux de combat et la marche sur Rome. Plus précisément, sur les années de la préparation et de l'accomplissement de la révolution fasciste : sans négliger l'expérience du conflit mondial et des luttes interventionnistes, les responsables décidèrent - en phase avec le sens donné au dixième anniversaire de la marche sur Rome (Decennale), dont l'Exposition de la Révolution était le point culminant - d'en réduire la place au profit de la commémoration de l'épopée de la prise du pouvoir. Les "réalisations» du régime connurent une restriction semblable, reléguées dans quelques salles, aux marges du trajet de l'exposition. Selon les directives de Mussolini, leur mise en scène aurait dû donner lieu à une nouvelle manifestation, qui ne fut néanmoins jamais réalisée.

$7 \mathrm{Au}$ début de 1932, commença la collecte de la documentation, en vue de laquelle coopérèrent le secrétariat général de l'exposition et les sections centrales et périphériques du Pnf, en sollicitant les prêts, les legs et la contribution des

[...] préfets, secrétaires fédéraux, secrétaires des Fasci, Podestà, dirigeants de toutes les organisations politiques et syndicales du fascisme, musées, bibliothèques, citoyens, autorités, des quadrumviri aux ministres, des sénateurs aux députés, aux

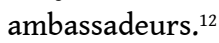

8 Dans les entrepôts bâtis en Via Cernaia commencèrent à arriver - de toute l'Italie et sous la supervision du bureau de collecte - des manuscrits, volumes, opuscules, papiers, tracts, manifestes, revues, journaux, photographies et reliques (des drapeaux aux armes) pour un total d'environ 15000 pièces. De sorte que l'Exposition de la Révolution peut être 
considérée, dès les mécanismes du repérage du matériel et des objets à exhiber, comme la réinterprétation fasciste de l'un des éléments centraux des processus de nation building inaugurés par les élites libérales au cours du XIX ${ }^{\mathrm{e}}$ siècle : le «monument » comme moyen d'autoreprésentation et d'autocélébration publique, et son édification comme entreprise visant à la construction des appartenances collectives ${ }^{13}$.

Pour respecter la date de l'inauguration, on procéda à la sélection des historiens et des artistes auxquels remettre la réalisation de la manifestation. Conformément à l'intention de "ressusciter l'atmosphère des années restituées » et d'éviter "l'aspect infécond, neutre, étranger qu'ont d'habitude les musées ", les organisateurs eurent recours à une technique d'exposition qui - sans sacrifier la dimension pédagogique véhiculée par la thèse : « les pages d'histoire écrites par les documents et par les reliques parleront plus que n'importe quel commentaire » - fit appel à l'art pour s'assurer l'implication émotive du spectateur. La préparation de chaque salle fut donc confiée à un historien, responsable du schéma narratif et du choix de la documentation et à un artiste, chargé du décor et des installations plastiques.

La très heureuse union entre art et histoire réalisée italianissimamente par l'Exposition du fascisme représentera peut être - affirmait Luigi Freddi dans le Guide historique - le début d'une révolution dans le domaine des musées et des expositions, et met au premier plan l'Italie dans un secteur où d'autres nations avaient beaucoup progressé et beaucoup réalisé. ${ }^{14}$

Ouvertes au public jusqu'à la fin d'octobre 1934, les salles de l'Exposition ${ }^{15}$ évoluaient selon un itinéraire obligé qui conduisait le visiteur, des démonstrations interventionnistes de mai 1914 à l'avènement du régime, à travers les années du conflit mondial, de l'après guerre et de la révolution fasciste. Elles reçurent quatre millions de visiteurs: un succès renforcé par les initiatives touristiques et de propagande qui en prolongèrent les effets bien au-dehors des murs du Palais des expositions, et qui les insérèrent dans le circuit des « temples de la foi du licteur $»^{16}$. Avant la fermeture, circula l'idée de convertir l'événement en exposition permanente, et d'instituer un Centre d'études sur le fascisme auquel confier la conservation, la mise en valeur et l'accroissement de la documentation recueillie. Vers la fin de l'année, le dessein de bâtir un nouveau siège ayant été abandonné ${ }^{17}$, les matériaux de l'exposition furent transférés dans la galerie nationale d'Art moderne de Rome où ils ne cessèrent pas de s'accroître grâce à la mobilisation du Pnf. En septembre 1937, tandis que l'exposition du Bimillénaire d'Auguste (Mostra Augustea della Romanità) ouvrait ses portes, l'Exposition de la Révolution connut sa deuxième version, caractérisée par l'aménagement de trois salles nouvelles - consacrées à l'Empire, à la Guerre d'Espagne et à Guglielmo Marconi - et par plusieurs remaniements de l'ancien décor, visant à exalter les liens avec l'histoire et avec la grandeur de la Rome impériale. Déclarée institution permanente en 1938, elle fut temporairement fermée en vue d'une réorganisation globale. Elle fut réouverte en 1939 et solennellement inaugurée dans sa troisième version le 28 octobre 1942, simultanément avec le Vicennale de la marche sur Rome. Malgré la présence d'un espace consacré au cinéma et au premier noyau du Centre d'études sur le fascisme (bibliothèque et archives), la manifestation ne répéta pas la réussite précédente, ce qui reflète le déclin de l'image consensuelle du régime au cours des années quarante. Et, comme pour appuyer ce déclin, après le 25 juillet 1943 les matériaux furent soustraits à la vue du public, retirés de Valle Giulia et emballés dans l'attente d'un nouveau lieu d'exposition. 
11 Pourtant, l'exposition ne se termina pas avec la chute du fascisme. Alors qu'une partie de la documentation suivit le régime en fuite au nord de l'Italie et qu'elle fut retrouvée - en 1945 - près du musée Lapidaire de Salò, la majorité des sources imprimées et des reliques demeurèrent dans les caves de la galerie nationale d'Art moderne et subirent maintes dispersions et pillages jusqu'à la Libération, où elles furent placées sous le patronage du Haut Commissariat pour les sanctions contre le fascisme. En vertu des accords signés par le commissaire liquidateur de l'exposition-Lucio Lombardo-Radice-et par les responsables des Archives nationales du royaume, les fonds de l'exposition et ceux du Centre d'études sur le fascisme furent à nouveau réunis et confiés aux Archives nationales, à la Bibliothèque nationale centrale et à la Bibliothèque d'histoire moderne et contemporaine de Rome, où ils sont toujours conservés à la suite d'un classement systématique qui a remis en cause la disposition originelle de la documentation.

Utilisés à plusieurs reprises par les historiens du fascisme - qui y ont puisé de précieux renseignements sur le fonctionnement $\mathrm{du}$ Pnf et sur les années du «fascismemouvement", et disposent aussi d'une collection presque complète de la presse du régime - les fonds archivistiques associés à l'exposition renvoient à un aspect négligé de son histoire ; aspect caché par la grandeur des dispositifs symboliques et rituels mis en place à l'occasion de sa réalisation. En effet, la provenance et la quantité du matériel archivistique - qui atteint aujourd'hui 300 boîtes $^{18}$ - témoignent de ce que l'Exposition de la Révolution fasciste exerça dès ses débuts une double fonction: d'un côté, l'autocélébration des origines subversives de la dictature, et leur inclusion dans les objets de culte de la religion politique fasciste ; d'un autre côté, la reconstitution de l'histoire de l'événement révolutionnaire et la promotion d'une révision de ses liens avec le passé libéral. Lieu de liturgie et de mémoire, le contexte d'exposition fut constamment associé à l'œuvre de "récolte » et de sédimentation des documents et des reliques. Si, dans l'état actuel de la recherche, il semble que le Centre d'études n'eut jamais le temps d'inaugurer ses activités, il est certain que la documentation recueillie pour l'exposition du Decennale fut cataloguée dès l'automne 1932, qu'elle s'accrut sans interruption durant les dix années qui suivirent et se transforma progressivement en pôle de conservation de fonds d'importance historique, parmi lesquels - à partir de 1939 - les papiers du Pnf.

13 Au lieu d'être en compétition, la dimension rituelle et l'œuvre de restitution du passé s'intégrèrent mutuellement et interagirent, au point que, à la commémoration de la révolution, ne tarda pas à se superposer celle de l'exposition chargée de sa représentation. On peut signaler, à cet égard, la méticulosité avec laquelle le Guide historique décrit l'organisation et la préparation de la manifestation: le lecteur y est informé des longues vicissitudes qui conduisirent à l'approbation et à la mise au point du projet d'exposition; des noms des collaborateurs et des détails techniques du décor, jusqu'aux mécanismes de fonctionnement du protocole d'enregistrement des documents et des objets reçus. On peut encore évoquer la publication, dans les premières pages du Guide, d'une série de photographies qui représentent - avec une surprenante sensibilité rétrospective - le backstage de l'exposition : un colis qui, visiblement, vient d'arriver, renfermant des reliques, des visions panoramiques des entrepôts de Via Cernaia, les échafaudages sur la façade du Palais des expositions, l'inspection des installations externes et l'évolution des travaux intérieurs, le montage des statues et des synthèses plastiques, on a même un instantané de la chute de l'échafaudage dans la salle de la marche sur Rome, comme s'il fallait témoigner jusqu'à la plus petite et à la plus dérisoire scène d'une entreprise qui aurait dû concourir à « donner au fascisme pleine conscience 
de son histoire $»^{19}$. Ce fut d'ailleurs sur la valeur de témoignage de l'expérience vécue que reposa la narration historique proposée par l'Exposition de la Révolution ; également sur le rôle des témoins qui, ayant expérimenté et mené la lutte pour la conquête du pouvoir, seraient les seuls à pouvoir en restituer la trame événementielle et les passions qui l'animèrent :

Nous, combattants encore vivants et armés de la Révolution fasciste, nous nous proclamons en droit de sculpter dans une forme éternelle, dans une matière perpétuelle, l'histoire de notre temps, dont nous fûmes et sommes les générateurs infatigables. Comme le soldat qui, face au foyer retrouvé raconte sa guerre, nous jetterons à l'avenir le récit de notre âpre vie, pour que les générations futures sachent qui nous fûmes, et comment et pourquoi nous luttâmes. Serons-nous capables de nous tourner en arrière et de regarder notre passé, pour le raconter sereinement avec des mots justes mais aussi de manière inexorablement vraie, et pour nous décrire tels que nous étions à l'époque ? Il le faut. Il est nécessaire que chacun de nous soit capable de devenir le descendant de lui-même. ${ }^{20}$

\section{« Déguiser, couvrir, cacher la vieille façade »}

La citation qui précède ne se borne pas à légitimer un récit historique entièrement fondé sur la mémoire des acteurs. "Regarder notre passé » et «nous décrire tels que nous étions à l'époque » en tournant le regard vers l'avenir, tout cela signifie associer à une attitude imprégnée d'historicisme à l'égard des expériences vécues une tentative de réinterprétation du présent qui renvoie à la dimension proprement visuelle de l'Exposition de la Révolution ; dimension visuelle dont le Guide de 1933 représente, encore une fois, un document précieux.

J'ai déjà fait allusion à la partition interne du guide, qui propose au lecteur une introduction historique à la période exposée, rédigée par Dino Alfieri et une description du parcours de l'exposition, signée par Luigi Freddi et enrichie par des photographies qui reproduisent - selon une technique et une mise en page qui auraient eu droit à une analyse à part ${ }^{21}$ - documents et vestiges, vues d'ensemble du montage et détails des synthèses plastiques, des photomontages, des installations et des interventions architecturales réalisés dans les locaux de l'exposition. Avant d'examiner la forme et le contenu de chaque salle, Freddi s'arrête sur les caractères de la façade, conçue par les architectes Adalberto Libera et Mario De Renzi afin de dissimuler, bien que temporairement, le siège de la manifestation : le Palais des expositions de Rome.

Quand il s'agit d'adapter à cet immeuble l'Exposition de la Révolution, se posa tout de suite le problème d'adapter l'extérieur, la façade, au caractère que monsieur Alfieri et ceux qui avaient été chargés de réaliser l'exposition, suivant les nettes dispositions du Duce, avaient l'intention d'imprimer au montage historicoartistique de l'intérieur. Une révolution qui avait marqué le début d'une ère nouvelle et, en tout cas, le détachement inexorable de tout un passé désormais et définitivement enterré, devait logiquement être représentée par des formes et par des aspects nouveaux et originaux qui puissent se différencier délibérément des tendances et des styles précédents. Ce complexe novateur et révolutionnaire ne pouvait naturellement pas se trouver emboîté dans une structure typiquement dixneuvième [...] comme l'était celle du Palais des expositions. Se posa alors le problème de la nouvelle façade, dont les termes se plaçaient entre la nécessité d'un motif modernissimo et l'obligation de faire des économies strictes, tout cela devant $s^{\prime}$ insérer dans le caractère obligatoire du temporaire. ${ }^{22}$ 
16

Se doter d'une façade nouvelle : une pratique qui résume presque à la lettre la fonction attribuée - à l'intérieur de l'exposition destinée à l'autoreprésentation des origines révolutionnaires de la dictature fasciste - aux images. Mais qui renferme aussi maintes traces de l'influence exercée sur l'utopie totalitaire, couramment synthétisée par la formule « homme nouveau », par l'idée de pouvoir modifier et interagir avec l'extérieur ; à savoir notamment, la manière selon laquelle les images furent considérées comme porteuses d'un pouvoir thaumaturgique et ré/générateur, véritable «matrice de l'histoire », « capable de produire un avenir sans précédent $»^{23}$.

peuvoir des images plonge ses propres racines dans le passé lointain de l'histoire de l'art ; on ne peut ici en retracer la généalogie, car il faudrait alors aborder, sur la longue période, la question de l'autorité de la création artistique et de ses liens avec les espaces et les langages du politique. Il me semble néanmoins nécessaire de rappeler le développement qu'un tel pouvoir reçut par le biais des idées utopistes de la deuxième moitié du XIX ${ }^{\mathrm{e}}$ siècle. Et, au XX⿳⺈冂大 siècle, par les mouvements avant-gardistes : c'est sur la fabrication des images que les artistes d'avant-garde établirent leurs prétentions de révolutionner, et de reconstruire, l'univers entier ${ }^{24}$; c'est d'ailleurs à la fabrication des images que ces artistes durent les rencontres les plus significatives avec la mentalité et les pratiques de l'avant-gardisme politique. Il suffit de rappeler, à cet égard, l'obstination avec laquelle les dictateurs - Benito Mussolini compris - s'auto-représentèrent en tant qu'artistes; ou encore, pour revenir à l'Italie des années trente et à l'opération de dissimulation accomplie par Libera et De Renzi sur la façade du Palais des expositions, les campagnes de rajeunissement urbanistique de la capitale, au cours desquelles alternèrent l'édification du visage monumental de la Rome fasciste et les démolitions, qui ne furent pas du tout temporaires, du centre historique ${ }^{25}$. Ce ne fut donc pas par hasard si - en vue de la construction de cet univers mythique et symbolique que fut l'Exposition de la Révolution fasciste-le choix des personnalités chargées du montage échappa partiellement aux dispositifs du recrutement culturel fasciste : à la présence de quelques jeunes talents issus des circuits de la consécration officielle, s'ajouta celle des personnalités les plus en vue des avant-gardes italiennes.

La coexistence - au sein de l'Exposition de 1932 - de différents mouvements d'avantgarde pourrait sembler tenir son origine de cette «tolérance esthétique » considérée par les historiens comme la base du mécénat poursuivi par la dictature dès l'avènement au pouvoir. Une tolérance esthétique qui s'avéra être une "astucieuse stratégie, parce qu'elle encouragea les différentes mouvances artistiques et intellectuelles à rivaliser pour la reconnaissance et la légitimation du gouvernement, et qu'elle autorisa ceux qui ne se reconnaissaient pas ouvertement dans le fascisme à participer aux initiatives publiques du régime $»^{26}$. Si l'on admet que les groupements d'avant-garde appartiennent au même pôle du champ artistique et littéraire, le modernisme, on peut néanmoins considérer la manifestation de 1932 comme l'une des rares circonstances où les responsables de la politique culturelle de la dictature eurent recours à une véritable tendance artistique afin de solliciter la création d'un « art proprement fasciste ». «L'Exposition de la Révolution fasciste marque le triomphe de l'art futuriste ", écrivit Filippo Tommaso Marinetti à l'occasion de son ouverture ${ }^{27}$, en soulignant - au-delà des différents groupes modernistes et malgré la rivalité qui existait parmi ses représentants - le caractère d'avant-garde de l'exposition, et sa victoire sur le classicisme vers lequel s'étaient orientées les options esthétiques officielles du fascisme.

Les Cahiers du Centre de Recherches Historiques, 31 | 2003 
L'identité avant-gardiste des artistes de la manifestation renvoie, en dernière analyse, à l'intention de présenter l'acte fondateur du régime comme une rupture. Rupture avec le passé libéral et - en même temps - avec le chaos et la désintégration de l'après guerre : l'itinéraire narratif de l'exposition fut expressément construit pour suggérer que l'aspiration à bouleverser le contexte politique et social italien était constamment associée à la promesse d'en reconstituer le fonctionnement et le sens, et pour attester que la charge subversive du fascisme était orientée, dès les débuts, vers le retour à l'ordre. Il suffit de se figurer - en faisant encore une fois référence aux reproductions publiées dans le Guide historique - les réactions des visiteurs contraints de se déplacer des salles grises de l'avant-guerre au dynamisme de celles consacrées à la lutte interventionniste et au premier conflit mondial; ou encore, de la violence et de l'agitation transmises par les salles de l'après-guerre à la majesté véhiculée par la salle de la marche sur Rome et par le corpus consacré au "charisme du chef », le «dux » guerrier, défenseur et incarnation de l'esprit de la nation. À l'intérieur des salles de l'Exposition de la Révolution fasciste, les origines révolutionnaires de la dictature atteignirent le statut de mythe moins par le fait qu'elles étaient reléguées dans un passé idéal et distancié, voire mythique, qu'en raison du fait que le présent - la stabilisation des débuts des années trente - était exhibé comme conséquence naturelle et cohérente d'une révolution dont les implications de classe et les autres potentialités étaient neutralisées. Et ce, en vertu de ce langage mythique qui - en préconisant une suspension du jugement rationnel pour un partage émotionnel, émotif, des concepts communiqués - aura été constamment employé par la dictature dans l'autoreprésentation de ses propres origines ainsi que de son propre futur ${ }^{28}$.

\section{« Mussolini et la Révolution »}

L'Exposition de la Révolution fasciste, synthèse vivante et palpitante de luttes, de sacrifices et de victoires, est la profession de foi que les anciens camarades offrent aux jeunes, afin que ces derniers, guidés par la lumière de nos martyrs et de nos héros, se préparent avec fierté à poursuivre le grand labeur. ${ }^{29}$

C'est par la publication de ce message de vœux de Mussolini, manuscrit sur papier à entête du Chef du Gouvernement et daté du 29 octobre - XI, que s'ouvre le Guide historique de l'Exposition de la Révolution fasciste. Une introduction qui n'a rien d'une coïncidence: tout comme le guide dans sa totalité, l'écriture de Mussolini dialogua au grand jour avec la structure de l'exposition, à partir du rez-de-chaussée et plus précisément des quinze salles consacrées à l'exhibition de la période comprise entre juillet 1914 et la marche sur Rome (salles A-Q). À l'intérieur des vitrines où étaient exposés- dans chaque local - les reliques et le matériel d'archives inhérents à la phase historique traitée, trouvèrent en effet leur place de nombreux documents dont Mussolini était l'auteur : des passages de son journal de guerre, les textes de ses discours dans les comices ainsi que de ses discours parlementaires; ses lettres et ses cartes postales; les articles rédigés durant sa longue carrière de journaliste; quelques manuscrits, pour ne s'en tenir qu'à l'essentiel d'une masse documentaire qui apportait le témoignage d'une militance exemplaire et de l'omniprésence du Duce au cours de la longue lutte pour la conquête du pouvoir. D'ailleurs, comme pour accentuer la valeur créatrice du verbe du chef, les mots de Mussolini débordaient des vitrines et ils intervenaient dans la dimension proprement artistique de la manifestation. Ainsi, ils étaient gravés sur les plaques en marbre disséminées tout au long du trajet de l'exposition; on les retrouvait, mêlés aux images et aux installations les plus diverses, au milieu des photomontages; reproduits dans des 
proportions gigantesques et élevés au rang de synthèses plastiques, ils s'étalaient sur les murs des salles, conformément au goût fasciste pour les sentences et pour les axiomes. Enfin, aux autographes de Mussolini, l'exposition de 1932 consacrait un local entier, la salle II située au premier étage ; étage du "fascisme-régime ", détaché du parcours de l'épopée révolutionnaire mais auquel on avait accès sitôt après le passage par la séquence monumentale, entièrement consacrée au chef et constituée par le salon d'honneur, la galerie des Faisceaux, la salle documentaire du Duce et le monument aux morts.

Dans cet espace sacré, auquel le visiteur accédait « en ayant encore, sous les yeux, la vision du drame vécu par notre pays dans les années de l'avant-guerre et de l'aprèsguerre" et à partir duquel « commençait la nouvelle histoire de l'Italie »" tout procédait du charisme du Duce. Tout concourait, de plus, à en édifier le culte: de la grande statue qui en reproduisait la figure jusqu'aux symboles romains du pouvoir fasciste; des vitrines qui donnaient à voir "quelques aspects singuliers et suggestifs de sa vie publique et privée » jusqu'à la réédification, à l'aide des objets originaux, des deux bureaux d'où il avait dirigé Il Popolo d'Italia ${ }^{31}$, partout, on était confronté à la mise en scène d'une personnalité qui se superposait - par sa seule présence et en vertu d'un passé où la multiplicité des fonctions exercées n'était pas occultée - à l'histoire fasciste, tout en garantissant la légitimité de ses origines révolutionnaires et la cohérence de son utopie.

Le fait que Mussolini constituait le fil rouge - et le centre - de l'Exposition de la Révolution fut d'ailleurs reconnu par les auteurs mêmes de la manifestation. Ils n'hésitèrent pas à remémorer l'engagement, personnel et constant, du Duce dans la réalisation de l'exposition, qui vit le jour «par la volonté du chef et par l'œuvre du Parti" "; ils en firent la source d'inspiration du travail accompli ; ils lui dédièrent l'effort collectif nécessaire à l'organisation de l'événement, sans omettre de spécifier que la « célébration des fastes de la Révolution fasciste » ne pouvait qu'être

[...] indissolublement liée à la pensée et à la volonté de Mussolini, que l'on retrouve tribun, combattant, agitateur, polémiste, meneur d'insurgés, chef du Gouvernement, harangueur et dominateur triomphant des fastes nationaux. ${ }^{32}$

Je ne peux analyser ici dans le détail la présence multiforme de Mussolini à l'intérieur de l'Exposition de la Révolution fasciste, la totalité de ses figurations - photographiques, picturales, plastiques, documentaires - comme l'ensemble des allusions métaphoriques au Duce du fascisme. Il suffit d'ailleurs de consulter le Guide de 1933, et de visionner les nombreuses représentations du chef qui y sont reproduites, pour s'apercevoir du fait que le Mussolini acteur de l'Exposition de la Révolution fut, fondamentalement, polysémique. Plus que la promotion et la victoire d'une image particulière, la manifestation décrétait l'interaction, la simultanéité et la coexistence de ces différents «mythes» de Mussolini "qui se manifestèrent à différentes périodes de sa vie, et qui tinrent leurs origines de milieux et situations politiques et culturelles divers $»^{33}$. Une coexistence confiée à la pluralité de langages employés dans le montage de l'exposition, et qui correspond à cette fonction de médiation perpétuelle entre les parties que les interprétations «polycratiques » de la souveraineté totalitaire ont l'habitude d'attribuer aux dictateurs fascistes.

24 En effet, les théories polycratiques ont été l'objet, durant les dix dernières années, d'une attention inédite de la part de l'historiographie du Ventennio ${ }^{34}$. Si leur adaptation au contexte italien a concouru à mettre en cause la conception d'un régime monolithique, à l'abri des conflits internes et entièrement géré par l'autorité des organismes centraux de l'État et du parti ; si, par conséquent, elles ont fait ressortir l'activité d'arbitrage menée 
par Mussolini au milieu des nombreux centres de pouvoir qui continuèrent à exister et à s'affronter jusqu'aux années quarante, leur application à la dimension symbolique peut contribuer à miner l'idée d'un chef figé et monumental, dont le charisme tirerait ses origines de la négation du passé révolutionnaire ainsi que de la dépersonnalisation de l'expérience vécue. En prenant en compte la totalité des systèmes linguistiques qui véhiculèrent - dans l'exposition de 1932 - l'icône du Duce, on s'aperçoit que le mythe suit et amplifie la nature polycratique du totalitarisme fasciste: sous forme de mots et d'images, Mussolini y fut représenté non seulement en tant que chef de la révolution mais encore de la nation, à savoir comme le conciliateur suprême de cette dialectique entre fascisation et italianisation qui traversa la dictature au cours de ces vingt années de détention du pouvoir.

\section{NOTES}

1. Pour une synthèse du débat historiographique sur le Troisième Reich : Ian Kershaw, The Nazi Dictatorschip : Problems and Perspectives of Interpretation, London, E. Arnold, 1993, 233 p.

2. Renzo De Felice, Mussolini il rivoluzionario. 1883-1920, Torino, Einaudi, 1965, 773 p. ; id., Mussolini il fascista, t. I, La Conquista del potere. 1921-1925 et t. II L'Organizzazione dello Stato fascista. 1925-1929, Torino, Einaudi, 1966-1968, 802 p. et 596 p. ; id., Mussolini il duce, t. I, Gli anni del consenso. 1929-1936 et t. II, Lo Stato totalitario. 1936-1940, Torino, Einaudi, 1974-1981, 945 p. et 840 p.; id., Mussolini l'alleato, vol. I, L'Italia in guerra. 1940-1943, t. I, Dalla guerra «breve» alla guerra lunga et t. II, Crisi e agonia del regime; vol. II, La Guerra civile. 1943-1945 (volume publié après le décès de l'auteur), Torino, Einaudi, 1990-1997, 1576 p. et 768 p.

3. Marcello Flores (ed.), Nazismo, fascismo, comunismo. Totalitarismi a confronto, Milano, Bruno Mondadori, 1998, 447 p. ; Marc Ferro (éd.), Nazisme et communisme. Deux régimes dans le siècle, Paris, Hachette, 1999, 278 p. ; Henry Rousso (éd.), Stalinisme et nazisme. Histoire et mémoire comparées, Paris, Complexe, 1999, 387 p. ; Enzo Traverso, Le Totalitarisme. Le XXe siècle en débat, Paris, Seuil, 2001, 923 p. Je ne peux pas fournir ici un panorama complet des évolutions de l'approche biographique des dictateurs fascistes; je voudrais pourtant signaler parmi les études les plus récentes : Renzo De Felice et Luigi Goglia, Mussolini. Il mito, Roma-Bari, Laterza, 1983, 317 p. ; Luisa Passerini, Mussolini immaginario. Storia di una biografia 1915-1939, Roma-Bari, Laterza, 1991, 290 p. ; Sergio Luzzatto, Il Corpo del duce. Un cadavere tra immaginazione, storia e memoria, Torino, Einaudi, 1998, 246 p. ; Pierre Milza, Mussolini, Paris, Fayard, 1999, 985 p. ; et, dans le contexte allemand, Ian Kershaw, Hitler, London-New York, Longman, 1991 ; id., Hitler, vol. I, 1886-1936 et vol. II, 1936-1945. Nemesis, London, The Penguin Press, 1998-2000, 845 p. et 1115 p.

4. Emilio Gentile, Le Religioni della politica. Fra democrazie e totalitarismi, Roma-Bari, Laterza, 2001, $250 \mathrm{p}$.

5. Simonetta Falasca-Zamponi, Fascist Spectacle. The Aesthetics of Power in Mussolini's Italy, Berkeley-Los Angeles-London, University of California Press, 1997, 293 p. ; Maria S. Stone, The Patron State. Culture \& Politics in Fascist Italy, Princeton, Princeton University Press, 1998, 334 p. ; Laura Malvano, Fascismo e politica dell'immagine, Torino, Bollati-Boringhieri, 1988, 195 p.

6. Je voudrais signaler que, dans la langue italienne, deux mots correspondent au français « exposition » : esposizione (lat. exponere) et mostra (lat. monstrare). Les deux mots sont presque synonymes, si ce n'est qu'au premier appartient la prérogative de signifier les expositions 
universelles, internationales ou nationales, et que le deuxième est normalement utilisé - à côté du nom de l'artiste ou du thème exposés - pour désigner une exposition artistique particulière. Sur l'Exposition de la Révolution fasciste voir, entre autres, Giorgio Ciucci, «L'autorappresentazione del fascismo. La mostra del decennale della marcia su Roma ", Rassegna di Architettura, 1982, p. 48-55 ; Partito nazionale fascista, Mostra della Rivoluzione fascista. Inventario, sous la direction de Gigliola Fioravanti, Roma, Pubblicazione degli Archivi di Stato, 1990, 360 p. ; Dante Ghirardo, "Architecte, Exhibitions, and the Politics of Culture in Fascist Italy ", Journal of Architectural Education, 1992, n 45, p. 67-75; Libero Andreotti, «The Aesthetics of War: The Exhibition of Fascist Revolution », ibid., p. 76-86 ; Jeffrey T. Schnapp, «Fascism's Museum in Motion », ibid., p. 87-97 ; id., Epic Demonstrations. Fascist Modernity and the 1932 Exhibition of the Fascist Revolution, Richard J. Golsan (ed.), Fascism, Aesthetics and Culture, Hanover, University Press of New England, 1992, 301 p., p. 1-37 ; Emilio Gentile, Il Culto del littorio. La sacralizzazione della politica nell'Italia fascista, Roma-Bari, Laterza, 1993, 326 p., p. 213-235 ; Maria S. Stone, «Staging Fascism. The Exhibition of the Fascist Revolution ", Journal of Contemporary History, 1993, n 28, p. 215-243 ; id., The Patron State, op. cit., p. 128-177 ; Antonella Russo, Il Fascismo in mostra, Roma, Editori Riuniti, 1999, 191 p. ; Paolo Morello, Fotomontaggio e rappresentazione politica alla mostra della Rivoluzione fascista, Sergio Bertelli (ed.), Il teatro del potere. Scenari e rappresentazioni del politico tra Otto e Novecento, Roma, Carocci, 2000, 245 p., p. 89-108 ; Claudio Fogu, «Fare la storia al presente. Il fascismo e la rappresentazione della Grande Guerra », Massimo Baioni e Claudio Fogu (ed.), « La Grande Guerra in vetrina. Mostre e Musei in Europa ", Memoria e Ricerca, 2001, n 7, p. 49-69.

7. Partito Nazionale fascista, Mostra della Rivoluzione fascista. Guida storica, sous la direction de Dino Alfieri et Luigi Freddi, Roma, Officine dell'Istituto d'Arti Grafiche di Bergamo, 1933, réimpression anastatique par l'hebdomadaire Candido Nuovo, Milano, Industrie Grafiche Italiane IGIS, 1982, 258 p.

8. Guida della mostra della Rivoluzione fascista, textes de Dino Alfieri et de Luigi Freddi, Firenze, Stabilimenti grafici di A. Vallecchi, 1932, 70 p. En 1935 fut en outre publié un répertoire des visites et des manifestations associées à l'exposition; Francesco Gargano, Italiani e stranieri alla mostra della Rivoluzione fascista, Roma, SAIE, 1935, $732 \mathrm{p}$.

9. L'histoire la plus complète de l'exposition reste, à présent, l'introduction de Gigliola Fioravanti in Partito nazionale fascista, Mostra della Rivoluzione fascista. Inventario, op. cit., p. 15-74.

10. À côté de Benito Mussolini - qui suivit personnellement l'Exposition de la Révolution dès ses origines et intervint à plusieurs reprises soit dans les travaux d'élaboration du schéma narratif soit dans le choix du décor - et de Dino Alfieri, siègeaient, dans le Comité de direction, les quadrumviri, le secrétaire et le secrétaire administratif du Pnf, le directeur du Popolo d'Italia, le secrétaire fédéral et le podestà de Milan.

11. Sur les évolutions du projet d'exposition et sur l'influence exercée par le directeur des Archives de la Guerre de Milan, Antonio Monti : Claudio Fogu, «Fare la storia al presente », op. cit. 12. Partito nazionale fascista, Mostra della Rivoluzione fascista. Guida storica, op. cit., p. 53.

13. George L. Mosse, The Nationalization of the Masses. Political Symbolism and Mass Movements in Germany from the Napoleonic Wars through the Third Reich, New York, Howard Ferting, 1974, 272 p. ; id., The Fascist Revolution. Toward a General Theory of Fascism, New York, Howard Ferting, 2000, $224 \mathrm{p}$.

14. Partito nazionale fascista, Mostra della Rivoluzione fascista. Guida storica, op. cit., p. 51. Les historiens cooptés dans la réalisation de l'exposition furent Dino Alfieri et Luigi Freddi, directeur et vice-directeur, Enrico Arrigotti, Giovanni Capodivacca, Dante Dini, Riccardo Gigante, Gigi Maino, Alessandro Melchiori, Antonio Monti, Piero Parini, Francesco Sacco. Parmi les artistes : Cipriano Efisio Oppo, dans la fonction de directeur artistique ; Antonio Barrera, Americo Bartoli, Arnaldo Carpanetti, Gherardo Dottori, Achille Funi, Adalberto Libera, Leo Longanesi, Mino Maccari, Giannino Marching, Guido Mauri, Publio Morbiducci, Marcello Nizzoli, Enrico 
Prampolini, Esodo Pratelli, Enrico Paolucci, Antonio Santagata, Mario Sironi, Giuseppe Terragni, Antonio Valente.

15. Rez-de-chaussée : salle A, De la conflagration européenne à la fondation du Popolo d'Italia (1914) [L. Freddi - E. Pratelli], salle B, Du rassemblement des Faisceaux d'action révolutionnaire à l'intervention de l'Italie dans la guerre européenne (1915) [L. Freddi-E. Pratelli]; salle C, La guerre italienne [A. Monti - A. Funi] ; salle D, La victoire italienne [A. Monti - A. Funi] ; salle E, De la victoire à la fondation des Faisceaux italiens de combat [G. Capodivacca - A. Carpanetti]; salles F-G, De la constitution des Faisceaux de combat à l'année 1919 [D. Dini - M. Nizzoli]; salles H-I, L'année 1920 [G. Maino - A. Bartoli, M. Maccari] ; salles L-M, Fiume et Dalmatie [R. Gigante - R. Marching] ; salle N, L'année 1921 [A. Melchiori-G. Mauri, E. Pratelli]; salle O, L'année 1920 jusqu'au début d'octobre [E. Arrigotti - G. Terragni] ; salle $\mathrm{P}$, Le rassemblement de Naples et les préliminaires de la marche sur Rome [F. Sacco - M. Sironi] ; salle Q, La marche sur Rome [F. Sacco - M. Sironi] ; salle R, Salon d'honneur [M. Sironi]; salle S, Galerie des Faisceaux [M. Sironi]; salle T, Salle documentaire du Duce [L. Longanesi]; salle U, Le monument aux martyrs [A. Valente, A. Libera] ; $1^{\mathrm{er}}$ étage : salle I, Faisceaux à l'étranger [P. Parini - P. Morbiducci] ; salle II, L'esprit. Bibliographie fasciste [A. Barrera, E. Paolucci] ; salles III, IV et V, Le travail dans le régime fasciste [A. Santagata, G. Dottori, E. Prampolini]. Faisaient en outre partie de l'exposition la Façade [A. Libera - M. De Renzi], le Hall et l'Entrée d'honneur. Pour la description de l'itinéraire historico-artistique: Partito Nazionale fascista, Mostra della Rivoluzione fascista. Guida storica, op. cit., p. 43-258 (texte et reproductions photographiques) et Luigi Freddi, Traccia storico-politica per la Mostra del fascismo, Roma, Tipografia della Camera dei Deputati, 1932, $104 \mathrm{p}$.

16. L'expression est employée par Emilio Gentile, Il Culto del littorio, op. cit., p. 196 et suivantes.

17. Je me réfère au projet de construction du palais du licteur, siège du directoire national du Pnf et de l'Exposition de la Révolution fasciste, sur la Via dell'Impero ; Maria Grazia Messina, «L'orma fermata nella pietra. Il concorso per il palazzo del Littorio del 1934 », Sergio Bertelli (ed.), Il Teatro del potere, op. cit., p. 109-147.

18. Partito nazionale fascista, Mostra della Rivoluzione fascista. Inventario, op. cit. Le Fonds Mostra est actuellement dispersé dans les séries suivantes : série Correspondance administrative (bb. 1-16) ; série Archives historiques de l'exposition [documentation du Parti national fasciste (bb. 17-59) et documentation diverse (bb. 59-87)] ; série Montage (bb. 88-96) ; série Exposition (bb. 97-173); série documentation photographique [Service photographique (bb. 174-201) et archives photographiques (bb. 202-300)] ; il faut ajouter une vaste collection de drapeaux et de reliques et les 3.000 volumes qui auraient dû constituer le début du Centre d'études sur le fascisme, dont la liste est publiée en annexe dans l'Inventaire du fonds.

19. Partito nazionale fascista, Mostra della Rivoluzione fascista. Guida storica, op. cit., p. 10-28. Dans l'impossibilité de publier les reproductions des photographies en question, je voudrais au moins mentionner le texte de leurs légendes : «Comment les vestiges arrivaient au bureau de collecte de Via Cernaia: la boîte dans laquelle étaient renfermés les vêtements ensanglantés du mort fasciste Pierino del Piano, et sur laquelle sa mère écrivit: 'Voilà les affaires de mon pauvre Piero' »; "Quelques aspects des entrepôts du bureau de collecte de Via Cernaia, où furent reçues, cataloguées, assurées, triées et coordonnées plus de dix-huit mille pièces"; "Le chantier improvisé de Via Nazionale vu du haut des échafaudages »; "La vieille façade du Palais des expositions est cachée derrière les échafaudages de la nouvelle et hardie façade de l'exposition »; «L'essai du positionnement d'un des grands faisceaux métalliques de 25 mètres de hauteur »; «Les travaux dans la salle de 1914 du peintre Pratelli : les vitrines et les murs attendent encore la vaste documentation qui complètera l'allure esthétique de l'ensemble »; « Morceau par morceau on monte la statue de 'L'Italie armée' du sculpteur Marini »; "Les silhouettes réalisées par le peintre Nizzoli pour la deuxième salle de 1919 ont encore un aspect fantomatique "; "Les décorations pleines de fantaisie de l'architecte Terragni pour la salle de 1922 durant le travail de réalisation "; "Les échafaudages de la salle de la marche sur Rome, conçue et réalisée par le 
peintre Sironi, tombent». Je voudrais enfin signaler qu'aux photographies du backstage sont associées les reproductions de deux manifestes de propagande de l'exposition, de quelques ébauches préparatoires et de quelques articles de Il Popolo d'Italia se référant à l'inauguration d'octobre 1932.

20. Partito nazionale fascista, Mostra della Rivoluzione fascista. Guida storica, op. cit., p. 45-46.

21. Les organisateurs de l'exposition firent appel - dans le but de laisser un témoignage sur les travaux préparatoires de la manifestation et de reproduire les matériaux nécessaires au montage - à la collaboration d'études photographiques de Rome et de Milan et, surtout, à la section photographique de l'Institut Luce. Je soumets à l'attention du lecteur un extrait du Guide historique, où Luigi Freddi souligne le rôle du Luce et la fonction reproductive assignée à la photographie; une fonction reproductive qui fut néanmoins démentie par l'usage, à l'intérieur des photomontages, des différents photogrammes : "Entretemps, on consultait des centaines de collections de journaux, et on en extrayait des passages documentaires. Mais du moment qu'on ne pouvait pas toujours découper les collections et que souvent certains documents étaient dans un tel état que l'exhibition en aurait compromis l'intégrité et la survivance, on eut recours aux reproductions qui furent exécutées en partie par la photographie, en partie par le système électrique 'Manophot' [...] à l'intérieur de deux locaux de Via Cernaia, fut improvisé un laboratoire photographique très spécialisé, équipé rapidement et selon une grande compétence par l'Institut national Luce, qui s'occupa aussi de son fonctionnement par l'intermédiaire de son personnel qualifié. Dans ce laboratoire, dans lequel on travaillait durant le mois d'octobre du matin au soir, furent pris 3127 reproductions photographiques, 2170 mètres carrés d'agrandissements faisant plus d'un mètre, 1030 photographies de formats compris entre 50 x 65 centimètres et $1 \times 1$ mètre, plus de 8000 photographies de formats compris entre $3 \times 18$ centimètres et $24 \times 30$ centimètres ", idem, p. 63. Sur la photographie dans le Ventennio fasciste : Carlo Bertelli e Giulio Bollati, L'Immagine fotografica. 1845-1945. Storia d'Italia, Annali 2, 2 vol., Torino, Einaudi, 1978, 202 p. et 308 p. ; Renzo De Felice e Luigi Goglia, Storia fotografica del fascismo, RomaBari, Laterza, 1981, 395 p. ; Ernesto G. Laura, Le Stagioni dell'aquila. Storia dell'Istituto Luce, Roma, Ente dello Spettacolo, 2000, 432 p. ; Sergio Luzzatto, L'Immagine del duce. Mussolini nelle fotografie dell'Istituto Luce, Roma, Editori Riuniti-Istituto Luce, 2001, 267 p.

22. Partito nazionale fascista, Mostra della Rivoluzione fascista. Guida storica, op. cit., p. 64-65. La façade de Libera et De Renzi consistait en un colossal cube rouge (trente mètres de chaque côté, pour recouvrir entièrement l'ancienne façade du Palais), sur lequel se profilaient quatre gigantesques faisceaux en métal de vingt-cinq mètres de hauteur, liés par un auvent où était posée l'inscription MOSTRA DELLA RIVOLUZIONE FASCISTA (un mètre et soixante de hauteur) ; sur la gauche et sur la droite des faisceaux, s'érigeaient deux immenses X (six mètres de hauteur), à savoir l'année dix de l'ère fasciste exprimée par le chiffre romain.

23. Éric Michaud, "La construction de l'image comme matrice de l'histoire ", Vingtième siècle, 2001, n. 72, p. 41-52; du même auteur et pour un approfondissement de la problématique en question : La Fin du salut par l'image, Nîmes, Éditions Jacqueline Chambon, 1992, 250 p. ; id., Un art de l'éternité. L'image et le temps du national-socialisme, Paris, Gallimard, 1996, 389 p. ; id., Fabriques de l'homme nouveau : de Léger à Mondrian, Paris, Carré, 1997, 120 p.

24. Je fais allusion au manifeste La Reconstruction futuriste de l'univers, signé par Giacomo Balla et Francesco Depero le 11 mars 1915; Giovanni Lista, Futurisme. Manifestes - Proclamations - Documentes, Lausanne, L'âge de l'homme, 1973, 450 p., p. 202-204.

25. Antonio Cederna, Mussolini urbanista. Lo sventramento di Roma negli anni del consenso, RomaBari, Laterza, 1979, 266 p. ; Italo Insolera, Roma moderna. Un secolo di storia urbanistica 1870-1970, Roma, Einaudi, $1993^{9}$, 345 p., p. 114-175 ; id., Roma fascista nelle fotografie dell'Istituto Luce. Con alcuni scritti di Antonio Cederna, Roma, Editori Riuniti-Istituto Luce, 2001, 271 p.; pour une récente relecture de la politique urbanistique fasciste, Vittorio Vidotto, Roma contemporanea, Roma-Bari, Laterza, 2001, 510 p., p. 171-224. 
26. Ruth Ben-Ghiat, La Cultura fascista, Bologna, il Mulino, 2000, 354 p., p. 20.

27. Filippo Tommaso Marinetti, «La Mostra della Rivoluzione fascista segna il trionfo dell'arte futurista », La Gazzetta del popolo, 29 octobre 1932.

28. Sur les «mythes modernes»: Roland Barthes, Mythologies, Paris, Éditions du Seuil, 1957, 233 p. ; Ernst Cassirer, "The Technique of Our Modern Political Myths », Symbol, Myth and Culture. Essays and Lectures of Ernst Cassirer 1935-1945, New Haven and London, Yale University Press, 1979, traduction italienne: «La tecnica dei nostri miti politici », Simbolo, mito e cultura, Roma-Bari, Laterza, 1985, 305 p., p. 246-270.

29. Partito nazionale fascista, Mostra della Rivoluzione fascista. Guida storica, op. cit., p. 2.

30. Partito nazionale fascista, Mostra della Rivoluzione fascista. Guida storica, op. cit., p. 210.

31. À l'intérieur de deux petites pièces insérées dans la séquence monumentale, furent en effet intégralement reconstitués les deux bureaux milanais du directeur de Il Popolo d'Italia: celui de Via Paolo di Cannobio, où Mussolini travailla de 1914 à 1920, et celui de Via Lovanio, où il s'installa de 1920 à 1922. La statue à laquelle j'ai fait référence fut réalisée par Mario Sironi, comme la galerie des Faisceaux et le salon d'honneur où elle était située. Cette statue représente l'une des rares figurations plastiques du Duce expressément réalisées pour l'Exposition de la Révolution. Cela ne signifie toutefois pas que les images de Mussolini présentées par la manifestation se limitèrent aux symboles abstraits et dépersonnalisés : son corps, et son visage, apparaissaient sur les nombreuses photographies - parmi lesquelles, un grand nombre de portraits qui le restituaient dans différentes circonstances et à des âges différents - étalées dans les vitrines et employées à l'intérieur des photomontages.

32. Partito nazionale fascista, Mostra della Rivoluzione fascista. Guida storica, op. cit., p. 9.

33. Emilio Gentile, Il Culto del littorio, op. cit., p. 264 et suivantes.

34. Salvatore Lupo, Il Fascismo. La politica in un regime totalitario, Roma, Donzelli, 2000, 456 p.; Emilio Gentile, La Via italiana al totalitarismo. Il partito e lo Stato nel regime fascista, Roma, Carocci, 2001, 321 p. Pour une récente lecture de l'ouvrage fondateur de la théorie policratique: Franz Neumann, Behemoth. The Structure and Practice of National-Socialism, New York, Oxford University Press, 1942, 429 p. ; « Lorenzo Ornaghi, Enzo Traverso, Mariuccia Salvati discutono Behemoth di Franz Neumann », Contemporanea. Rivista di storia dell' 800 e del '900, juillet 2002, nº 3, p. 519-536.

\section{AUTEUR}

\section{MADDALENA CARLI}

EHESS, doctorante 